\title{
Medication errors in the neonatal intensive care unit: special patients, unique issues
}

\section{J E Gray, D A Goldmann}

\section{Medication errors are quite common in the neonatal intensive care unit}

M edical errors are a common occurrence in the neonatal intensive care unit (NICU). Although this high risk, fragile patient population is prone to a wide array of errors, medication errors are particularly common. Medication errors were the most common error type submitted to the Vermont Oxford Network's NICQ.org voluntary reporting system. ${ }^{1}$ Kaushal and colleagues $^{2}$ identified errors in $5.5 \%$ of NICU medication orders. Of note, potential adverse drug events (errors that had the potential to harm the patient but were intercepted, or potentially harmful errors that reached the patient but fortuitously did not result in injury) occurred eight times more often in NICU patients than in adults in hospital. Neonates, especially very low birthweight babies, are particularly vulnerable to adverse sequelae of medication errors as they have a limited ability to "buffer" such mistakes.

Nursing practice has long recognised the need for extreme vigilance and a structured approach to preventing medication errors. The five "Rights" provide a framework for improving medication safety in nursing. These basic principles of standard operating procedure try to address all of the steps in the medication process: ordering, dispensing, administering, and monitoring drugs. Nurses attempt to ensure that the Right drug is given in the Right dose at the Right interval via the Right route to the Right patient.

Although nurses focus on providing error-free care, research into human factors teaches us that dedication, training, and vigilance are not enough to prevent errors in complex systems. ${ }^{3}{ }^{4}$ Error prevention must be a multidisciplinary process, involving doctors, pharmacists, and nurses working as a team. The team must be backed up by robust healthcare delivery systems operating in a "culture of safety", providing staff with a working environment that provides safeguards against human fallibility. Nowhere in the hospital is the challenge greater than in the NICU.
The repertoire of drugs prescribed in the NICU is relatively limited compared with adult and older paediatric populations, but the process of ordering, dispensing, and administering them is more complex in newborns. The process for ordering drugs in the NICU is uniquely complex; more than three quarters of medication errors occurred during this stage. ${ }^{2}$ As doses are calculated according to the infant's weight, virtually all prescriptions require patient specific calculations and may need to be updated as the infant gains or loses weight. Weight and gestational age are not the only factors that need to be considered. For premature infants, doses must also be modified on the basis of the developmental maturity of specific metabolic and excretory pathways.

Drugs prescribed in the NICU are often used in an off label or unlicensed fashion. ${ }^{5}$ As a result, no comprehensive and authoritative standards for doses exist. Therefore clinicians are often confronted by a dizzying array of published reference standards for a single drug. Recommendations are surprisingly variable even for drugs that have been studied in neonates and approved for use by the Food and Drug Administration. For example, widely used references in the United States suggest total daily ampicillin doses that vary by a factor of 3-4 for the same $1 \mathrm{~kg}$ patient. ${ }^{6-8}$ Certainly, for a drug with a wide therapeutic index, this difference may not be clinically significant. However, the lack of a single dosing standard within a hospital can complicate the development of error reduction strategies in which doctors, nurses, and pharmacists verify doses.

NICU drug dispensing is also complex. Pharmacists often have to dilute stock solutions in order to provide doses that are extremely minute compared with adult standards.' In this issue of the journal, Chappell and Newman ${ }^{10}$ document the potential for 10-100-fold dosing errors associated with the use of stock drug concentrations intended primarily for use in adults. Of particular concern is the fact that three of 10 drugs at risk of 10-fold dosing errors and all four at risk of 100-fold errors are high alert drugs as defined by the Institute for Safe Medication Practice. ${ }^{11}$ Even more alarming is the fact that these decimal point errors represent only a portion of the calculation errors that can complicate the ordering and preparation process.

Errors in the route of administration of drugs and enteral nutrition are also common, complicated $13.3 \%$ of potentially harmful medication errors seen in two NICUs in the United States. ${ }^{12}$ In another report, Suresh and colleagues ${ }^{1}$ noted potentially very serious administration errors, such as infants fed expressed breast milk intravenously. Unlike adult care units, enteral feeding tubes and intravenous lines are often of the same calibre and appearance and have hubs of similar size. This type of error could be prevented by adopting administration systems with "forcing functions" that prevent feeding pumps and syringes from being attached to intravenous lines. Regrettably, these systems are not in widespread use in NICUs, in part because of incompatibilities with existing equipment and work flow processes.

Finally, patient misidentification occurs commonly in the NICU. One quarter of the serious medication errors reported in this issue by Simpson et $\mathrm{al}^{13}$ involved patient misidentification. Similarly, Suresh et al found that $11 \%$ of NICU errors involved misidentification. The increasing incidence of multiple gestations with premature births is in part responsible for these errors, but suboptimal systems for identifying babies contribute to the problem. Analyses by the Center for Patient Safety in NICU care suggest that as many as one half of infants in the NICU are at risk of misidentification on any given day (unpublished work).

The patient safety movement has highlighted numerous approaches to preventing medication errors, but which interventions have the potential to have the greatest impact? Fortescue and colleagues $^{12}$ have identified three interventions with the largest potential to decrease NICU medication errors: ward based clinical pharmacists, computerised physician order entry (CPOE), and improved communication among NICU clinicians.

The involvement of clinical pharmacists in intensive care unit rounds significantly reduces dosing and other types of error in adult care. ${ }^{14}$ In this issue, Simpson et al ${ }^{13}$ conclude that similar improvements can be achieved through the input of an NICU based clinical pharmacist. Although their data are encouraging, confidence in their 
conclusion must be tempered by several methodological concerns. Multiple interventions were applied during the study, and the exact timing and interaction of these interventions are unclear. Some discussion of the background and expertise of the pharmacists participating in the intervention would have been valuable as neonatal expertise and experience are almost certainly important. Unfortunately, the authors expressed the major outcome measure as the absolute number of medication errors, rather than error rates per number of patient days or per number of orders written. We hope that these important denominators remained relatively stable during the study period. In addition, it is unclear to what extent the ascertainment methods used, which relied on voluntary reporting by clinicians, were accurate and unbiased. Voluntary reporting, although valuable on many levels, cannot be relied on to provide accurate incidence data. Finally, the authors provide no statistical measures of differences between the periods before and after intervention.

Implementation of CPOE in the NICU presents special challenges. Systems designed for use in older patients may not adequately address the unique aspects of NICU medication ordering. Unfortunately, development of systems appropriate for use in paediatric and neonatal patients has lagged. Industry must be challenged to provide software applications that are appropriate for NICUs. CPOE almost certainly will have to be integrated with other hospital clinical information systems to have maximum impact on error prevention. Adequate, built in decision support, using population specific knowledge bases, is essential for detecting drug interactions, out of range doses, and other prescribing problems. The LeapFrog Group, ${ }^{15}$ a consortium of Fortune 500 companies, has urged hospitals in the United States to adopt CPOE. Given Leapfrog's leverage and influence, recognition of the unique needs of NICUs would be welcome.
Where CPOE is not available, attention to good prescribing practices and accurate communication are essential. $^{5}{ }^{16}$ This is true not only for written orders, but verbal ones as well. The process for verbal orders should include a system of "read back" verification to ensure accuracy. Lacking CPOE, clinicians (doctors, nurses, and pharmacists) must implement unambiguous guidelines on appropriate dosing for NICU patients. Good communication and teamwork requires a blame free environment and a culture that places a high value on reporting and discussing patient safety concerns and systems problems.

Finally, NICU clinicians must remain aware of the advances in patient safety made in other industries. Crew Resource Management, which has been pivotal to improving the safety record of the aviation industry, may be particularly useful in helping teams communicate effectively and safely. ${ }^{17}$ Translation of technologies from the retail sector, such as bar coding and radio frequency identification, may be helpful in preventing patient misidentification. When feasible, engineering approaches using affordances and reminders, forcing functions, and constraints may help staff to avoid errors due to human factors. Of course, these novel approaches to creating a safe care environment will have to be tailored to the very special and challenging environment of the NICU.

Arch Dis Child Fetal Neonatal Ed 2004;89:F472-F473.

doi: $10.1136 /$ adc. 2003.046060

\section{Authors' affiliations}

D A Goldmann, Department of Medicine and Quality Improvement Program, Children's Hospital, Boston, MA 02115, USA

J E Gray, Department of Neonatology, Beth Israel Deaconess Medical Center, Boston, MA 02115, USA

Correspondence to: Dr Gray, Department of Neonatology, Beth Israel Deaconess Medical Center, Boston, MA 02115, USA: igray@bidmc.harvard.edu
Accepted 19 December 2003

Supported in part by a grant from the Agency for Healthcare Research and Quality (funded by AHRQ P2O HS 11583).

\section{REFERENCES}

1 Suresh G, Horbar JD, Plsek P, et al. Voluntary anonymous reporting of medical errors for neonatal intensive care. Pediatrics 2004;113:1609-18.

2 Kaushal R, Bates DW, Landrigan C, et al. Medication errors and adverse drug events in pediatric inpatients. JAMA 2001;285:2114-20.

3 Reason JT. Managing the risks of organizational accidents. Brookfield, VT: Ashgate Publishing Company, 1997.

4 To err is human: building a safer health system. Washington, DC: National Academy Press, 2000.

5 O'Donnell CP, Stone RJ, Morley CJ. Unlicensed and off-label drug use in an Australian neonata intensive care unit. Pediatrics 2002;110. http:// www.pediatrics.org/cgi/content/full/110/5/ e52.

6 Avery GB, Fletcher MA, MacDonald MC, eds. Neonatology: pathophysiology and management of the newborn. Philadelphia: Lippincott Williams and Wilkins, 1999

7 Young TE, Mangum B. Neofax. Raleigh, NC: Acorn Publishing, 2003

8 American Academy of Pediatrics. Red book: 2003 report of the Committee on Infectious Diseases. Washington DC: American Academy of Pediatrics, 2003.

9 Koren G, Haslam RH. Pediatric medication errors: predicting and preventing tenfold disasters. J Clin Pharmacol 1994:34:1043-5.

10 Chappell K, Newman C. Potential tenfold drug overdoses on a neonatal unit. Arch Dis Child Fetal Neonatal Ed 2004;89.

11 http://www.ismp.org/Survey/NewsLetter/ Survey20030821.asp. Accessed 15 December, 2003.

12 Fortescue EB, Kaushal R, Landrigan CP, et al. Prioritizing strategies for preventing medication errors and adverse drug events in pediatric inpatients. Pediatrics 2003;111:722-9.

13 Simpson JH, Lynch R, Grant J, et al. Reducing medication errors in the neonatal intensive care unit. Arch Dis Child Fetal Neonatal Ed 2004:89.

14 Leape LL, Cullen DJ, Clapp MD, et al. Pharmacist participation on physician rounds and adverse drug events in the intensive care unit. JAMA 1999;282:267-70.

15 http://www.leapfrog group, org. Accessed 15 December 2003.

16 Joint Commission on Accreditation of Healthcare Organizations. 2004 National Patient Safety Goals. http://www.jcaho.org/ accredited+organizations/patient+safety/ 04+npsg/04_npsg.htm. Accessed 15 December 2003.

17 Pizzi L, Goldfarb NI, Nash DB. Crew resource management and its applications in medicine. In: Making healthcare safer. Rockville, MD: Agency for Healthcare Research and Quality, 2002.

that taurine deficiency in cats was associated with retinal degeneration, which was reversed by taurine supplementation. ${ }^{2}$ This observation coupled with the high concentration of taurine in the developing brain ${ }^{3}$ and mature retina ${ }^{4}$ raised suspicion that taurine may play an important role in brain development. This was supported by observations that brain taurine concentration of several species decreased during the weaning period $^{3}$ and that taurine was the primary free amino acid in the milk of most mammals, including humans. ${ }^{5}$ Moreover, labelled taurine injected intraperitoneally into lactating rats was found in the milk 
of the dam as well as the brain of the suckling pups, ${ }^{6}$ suggesting that adequate intake of taurine was important for maintaining brain taurine content.

Shortly after the observation that taurine deficiency in cats resulted in retinal degeneration, evidence that taurine may be a conditionally essential nutrient for the human infant began appearing. The first such evidence came from a study in Scandinavia showing that plasma and urinary taurine concentrations of formula fed infants were lower than those of infants fed human milk, ${ }^{7}$ whereas the plasma and urinary concentrations of all other amino acids were higher in formula fed infants. ${ }^{89}$ This was attributed to the presence of taurine in human milk but not formulas. Subsequently, it was shown that prolonged taurine-free parenteral nutrition resulted in retinal degeneration that was reversed with taurine supplementation. ${ }^{10}$ Retinal abnormalities were also found in primates fed a taurine-free infant formula. ${ }^{11}$

On the basis of these findings, taurine was added to most infant formulas by the early to mid 1980s. The only randomised controlled trial of taurine supplementation was started before its routine addition to formulas but terminated for ethical reasons after 37 rather than the planned 50 infants were enrolled. Nonetheless, preterm infants assigned to the taurine supplemented formula had a more mature auditory brain stem evoked response than those assigned to the taurine-free formula. ${ }^{12}$ However, no differences in electroretinograms or Brazelton scores were detected. Infants fed taurine supplemented formulas also have a bile salt conjugation pattern more like that of breast fed infants as well as a larger bile salt pool, but reported effects on fat absorption have been mixed. ${ }^{13-15}$

Owing to the relative lack of evidence that taurine supplementation of infant formulas has beneficial clinical effects, recent recommendations for the nutrient contents of term infant formulas do not include a minimum content of taurine. ${ }^{16}$ However, as formulas have contained taurine for almost two decades and these seem to be well tolerated, a maximum amount $(12 \mathrm{mg} / 100$ kcal) is specified. This is near the maximum content observed in human milk and about 25\% more than the content of modern formulas. A minimum content of taurine $(5 \mathrm{mg} / 100 \mathrm{kcal})$ is specified for preterm infant formulas but without much enthusiasm. ${ }^{17}$

The findings of Wharton et al, ${ }^{18}$ reported in this issue, suggest that the recommendations for taurine content of infant formulas should be reconsidered. These findings suggest that low plasma taurine concentration during the hospital stay may explain the paradox of higher developmental scores at 18 months $^{19}$ and 7 years of age ${ }^{20}$ in preterm infants assigned to a nutrient enriched compared with a term formula during initial hospital admission but similar scores in infants assigned to banked human milk compared with the nutrient enriched formula despite the fact that the nutrient density of the banked human milk was even lower than that of the term formula. ${ }^{21}$ Although the possibility that the paradoxical neurodevelopmental outcomes were related to taurine intake during infancy was suggested in reviews by Sturman and Chesney in $1995^{22}$ and Chesney et al in $1998,{ }^{23}$ Wharton et al ${ }^{18}$ provide the first indication that this explanation may be valid. They show that the Bayley mental developmental index at 18 months of age and the WISC-R arithmetic subtest score at 7 years of age are correlated with plasma taurine concentrations during infancy. They also report that the positive association of neurodevelopment with own mother' milk ${ }^{24}$ was not significant after plasma taurine concentration had been allowed for. These findings are attributed to the presence of taurine in the preterm formula and human milk but not in the term formula.

As the authors emphasise, these findings are far from robust. Firstly, they are not derived from a randomised, controlled trial but, rather, from a retrospective analysis of existing data. Secondly, the strength of the reported relations is modest ( $r=0.28$ and 0.22 ). Nonetheless, they support the hypothesis that low neonatal taurine status adversely affects later neurodevelopment of preterm infants and that the neurodevelopmental advantage of human milk may be related to its taurine content. Thus the new data provide further support for the view that taurine is a conditionally essential nutrient for the preterm infant. They also provide an additional example of apparent long term effects of short term early differences in nutrient intake.

The findings of Wharton et al also present a quandary. Randomised, controlled trials of taurine supplementation for both preterm and term infants should clearly be the next step, but would either trial now be ethical? Like so many other issues in neonatal nutrition and, indeed, all of clinical medicine, it is unlikely that the role of taurine in infant nutrition will ever be evaluated in a randomised controlled trial.

Arch Dis Child Fetal Neonatal Ed 2004;89:F473-F474.

doi: $10.1136 /$ adc. 2004.055095
Correspondence to: Dr Heird, Children's Nutrition Research Center, Department of Pediatrics, Baylor College of Medicine, 1100 Bates Street, Houston, TX 77030, USA; wheird@bcm.tmc.edu

\section{REFERENCES}

1 Tiedmann F, Gmelin L. Binige nede bestandtheile der galle des ochsen. Annalen der Physik und Chemi 1827:9:326.

2 Hayes KC, Carey RE. Retinal degeneration associated with taurine deficiency in the cat. Science 1975;188:949-51.

3 Sturman JA, Gaull GE. Taurine in the brain and liver of the developing human and monkey. J Neurochem 1975:25:831-5.

4 Pasantes-Morales $\mathrm{H}$, Klethi $\mathrm{H}$, Ledig M, et al. Free amino acids of chicken and rat retina. Brain Res 1972;41:494-7.

5 Rassin DK, Sturman JA, Gaull GE. Taurine and other free amino acids in milk of man and other mammals. Early Hum Dev 1978;2:1-13.

6 Struman JA, Rassin DK, Gaull GE. Taurine in developing rat brain: transfer of $\left[{ }^{35}\right.$ S]taurine to pups via the milk. Pediatr Res 1977;1 1:28-33.

7 Gaull GE, Rassin DK, Räihä NCR, et al. Milk protein quantity and quality in low-birthweight infants. III. Effects on sulfur amino acids in plasma and urine. J Pediatr 1977:90:348-55.

8 Rassin DK, Gaull GE, Heinonen K, et al. Milk protein quantity and quality in low-birth-weight infants. II. Effects on selected aliphatic amino acids in plasma and urine. Pediatrics 1977:59:407-22.

9 Rassin DK, Gaull GE, Heinonen K, et al. Milk protein quantity and quality in low-birth-weight infants. IV. Effects on tyrosine and phenylalanin in plasma and urine. J Pediatr 1977;90:356-60.

10 Geggel H, Ament $M$, Heckenlively J. Nutritional requirement for taurine in patients receiving longterm, parenteral nutrition. $N$ Engl J Med 1985;312:142-6.

11 Sturman JA, Wen GY, Wisniewski HM, et al. Retinal degeneration in primates raised on a synthetic human infant formula. Int J Dev Neurosci 1984;2:121-30.

12 Tyson JF, Lasky R, Flood D, et al. Randomized trial of taurine supplementation for infants $\leqslant$ 1,300-gram birth weight: effect on auditory brainstem-evoked responses. Pediatrics 1989;83:406-15.

13 Okamoto E, Rassin DK, Zucker CL, et al. Role of taurine in feeding the low-birth-weight infant. J Pediatr 1984; 104:936-40.

14 Jarvenpaa A-L, Rassin DK, Kuitunen P, et al. Feeding the low-birth-weight infant. III. Diet influences bile acid metabolism. Pediatrics 1983;72:677-83

15 Galeano NF, Darling P, Lepage G, et al. Taurine supplementation of premature formula improves fat absorption in preterm infants. Pediatr Res 1987:22:67-71.

16 Raiten DJ, Talbot JM, Waters JH. Assessment of nutrient requirements for infant formulas. J Nutr 1998; 128:2059S-293S.

17 Klein CJ. Nutrient requirements for preterm infant formulas. J Nutr 2002;132:1395S-577S.

18 Wharton BA, Marley R, Isaacs EB, et al. Low plasma taurine and infant development. Arch Dis Child Fetal Neonatal Ed 2004;89.

19 Lucas A, Morley R, Cole TJ, et al. Early diet in preterm babies and developmental status at 18 months. Lancet 1990:335:1477-81.

20 Lucas A, Morley R, Cole TJ. Randomised trial of early diet in preterm babies and later intelligence quotient. BMJ 1998;317:1481-7.

21 Lucas A, Morley R, Cole TJ, et al. A randomised multicenter study of human milk versus formula and later development in preterm infants. Arch Dis Child Fetal Neonatal Ed 1994;70:F141-6.

22 Sturman JA, Chesney RW. Taurine in pediatric nutrition. In: Gaul GE, eds. Pediatr Clin North Am 1995;42:879-97.

23 Chesney RW, Helms RA, Christensen M, et al. The role of taurine in infant nutrition. Adv Exp Med Biol 1998:442:363-76.

24 Morley R, Cole TH, Powell R, et al. Mother's choice to provide breast milk and developmental outcome. Arch Dis Child 1988;63:1385. 